\title{
Support Vector Machines for Improving Vehicle Localization in Urban Canyons
}

\author{
Guermah Bassma ${ }^{1, *}$, El Ghazi Hassan ${ }^{1, * *}$, and Sadiki Tayeb $^{2, * * *}$ \\ ${ }^{1}$ STRS Laboratory, National Institute of Posts and Telecommunication, Rabat, Morocco \\ ${ }^{2}$ Université Internationale de Rabat (UIR), Faculty of Computing and Logistics, TICLab, Sala El Jadida, Morocco
}

\begin{abstract}
Since the middle ages, the need to identify the vehicles position in their local environment has always been a necessity and a challenge. Today, GNSS-based positioning systems have penetrated several field, such as land transport, emergency systems and civil aviation requiring high positioning accuracy. However, the performances of GNSS-based systems can be degraded in harsh environment due to non-line-of-sight (NLOS), Multipath and masking effects. In this paper, for improving vehicle localization in urban canyons, we address a very challenging problem related to GNSS signal reception state detection (LOS, NLOS or Multipath). A SVMbased system for GNSS Multipath detection using the fusion of information provided by two GNSS antennas is proposed. In this work, we aim to explore the potential of machine learning, and more precisely, Support Vector Machines (SVM) to identify GNSS signals reception state. The SVM-based system developed in this work has used the $\mathrm{C} / \mathrm{NO}$ of signals provided by RHCP and LHCP antennas, and satellite elevation as classification criteria. The training data set is constructed by several experimental studies done in real environments, Calais, France . Furthermore, four SVM kernel functions are tested, namely, Linear, Gaussian, Cubic and Quadratic.

A GNSS signal reception state detection by applying the proposed SVM-based classifier is demonstrated on real GPS signals, and the efficiency of the system is shown. We obtain empirically an accuracy of signal detection about $93 \%$.
\end{abstract}

\section{Introduction}

In harsh environment, the performances of GNSS-based positioning systems can be strongly affected, due to Multipath effects. For improving navigation performance, several solutions are proposed in the literature to filter, mitigate, eliminate, detect and modeling Multipath effects [1$8]$.

In this paper, the motivating problem is to detect the GNSS signals reception state. In [9] the authors are used the C/NO ratio to detect the Multipath signals. However, the performance of $\mathrm{C} / \mathrm{N} 0$ ratio used as indicator for Multipath detection still limited and imprecise. Other investigations have shown that fuzzy logic approach can be applied to classify GPS data based on signal degradation conditions [10-13]. In [10], a GPS-code-based measurements fuzzy processing is applied to detect the optimal observables, using the GDOP (Geometrical Dilution Of Precision) parameter and the signal-to-noise ratio (SNR). Others works have applied fuzzy logic to detect the signal quality using $\mathrm{C} / \mathrm{N} 0$ and the dilution-of-precision (DOP) [12-14].

In [15], the fuzzy logic approach is applied to classify the data quality using the DOP value and the difference between the measured and excepted $\mathrm{C} / \mathrm{N} 0$ as input fuzzy variables.

\footnotetext{
*e-mail: bassmaguermah@gmail.com

**e-mail: elghazi@inpt.ac.ma

***e-mail: tayeb.sadiki@uir.ac.ma
}

In other study [16], the authors have used the data fusion provided by dual-polarisation antenna for NLOS and Multipath detection basing on C/NO. Other approach is proposed by Monask Socharoentum in [17], it's based on Nav2Nav (Navigation-to-Navigation) technique in which pseudo-range corrections and machine learning work together to detect GNSS Multipath signals.

In this study, we have exploited the potential of the RHCP and LHCP to process the GNSS signal. we have proposed a new classifier of GNSS signals, based on a very robust technique of Machine learning called SVM [18][19]. The main idea of this system is to train the data set of real observations constructed from experimental studies to learn a model able to be used for predicting the GNSS signal reception state in real scenario.

A signal reception state detection by applying the proposed classifier system is demonstrated on real GPS signals, and the efficiency of the classifier is shown.

\section{Background}

\subsection{Support Vector Machines (SVM)}

In machine learning, support vector machine, also called support vector network, is a supervised learning technique that performs classification by finding the hyperplane that maximizes the margin between the two classes [20].

The main steps of SVM algorithm is presented as follows (Algorithm (1)): 


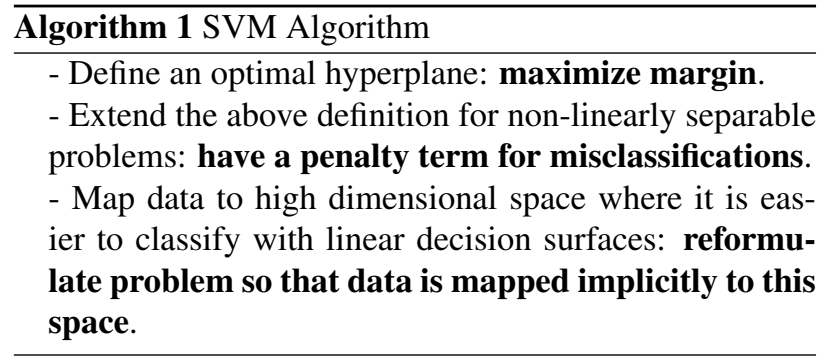

\subsubsection{Linear SVM}

To classify our data set, several hyperplane are present. SVM works to select the separating hyperplane that maximizes the margin (See figure 1).
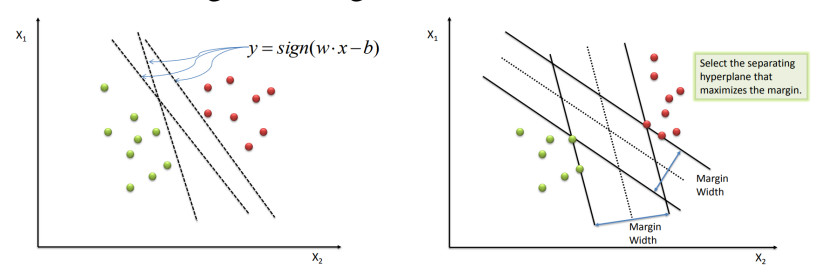

Figure 1. SVM Training.

The vectors that define the hyperplane are called the support vectors. Furthermore, to define an optimal hyperplane we need to maximize the width of the margin $(w)$, which can be presented as :

$$
\max \frac{2}{\|w\|}
$$

Where :

$$
\begin{gathered}
(w x+b) \geqslant 1, \forall x \text { of class } 1 \\
(w x+b) \leqslant-1, \forall x \text { of class } 2
\end{gathered}
$$

The optimization problem can be defined by the following system :

$$
\left\{\begin{array}{c}
\min \frac{1}{2}\|w\|^{2} \\
y_{i}\left(w x_{i}+b\right) \geqslant 1, \forall x_{i}
\end{array}\right.
$$

We find $w$ and $b$ by solving the system 2 using Quadratic Programming [21]. If the data is linearly separable, there is a unique global minimum value. However, if the data is not linearly separable, the system becomes non-solvable. Furthermore, the SVM technique consists to search a hyperplane that completely separates the vectors into two non-overlapping classes. However, some data can be not perfectly separable. In this case, SVM aims to find the hyperplane that maximizes the margin and minimizes the misclassifications.

The SVM algorithm aims to maintain the slack variable $\xi_{i}$ to zero while maximizing margin. We note that the SVM algorithm does not minimize the number of misclassifications but the sum of distances from the margin hyperplanes.

In this case, the optimization problem (system 2) can be reformulated as follows :

$$
\left\{\begin{array}{c}
\min \frac{1}{2}\|w\|^{2}+C \sum_{i} \xi_{i} \\
y_{i}\left(w x_{i}+b\right) \geqslant 1-\xi_{i}, \forall x_{i} \\
\xi_{i} \geqslant 0
\end{array}\right.
$$

Where $C$ trades-off margin width and misclassifications.

\subsubsection{Non-Linear SVM}

To separate two groups, the simplest way is with a straight line (1 dimension), flat plane (2 dimensions) or an N-dimensional hyperplane. However, in some case, a nonlinear region can separate the data more efficiently. So, where a linear hyperplane cannot be used to separate the two groups, the SVM uses two solutions, namely, "kernel function" or "Mapping Data to a High-Dimensional Space", to make data possible to perform the linear separation.

\section{Mapping Data to a High-Dimensional Space :}

To map data into new space, we must find function $\phi(x)$. The SVM formulation becomes :

$$
\left\{\begin{array}{c}
\min \frac{1}{2}\|w\|^{2}+C \sum_{i} \xi_{i} \\
y_{i}(w \phi(x)+b) \geqslant 1-\xi_{i}, \forall x_{i} \\
\xi_{i} \geqslant 0
\end{array}\right.
$$

Figure 2 illustrates the linear classifiers in highdimensional spaces.

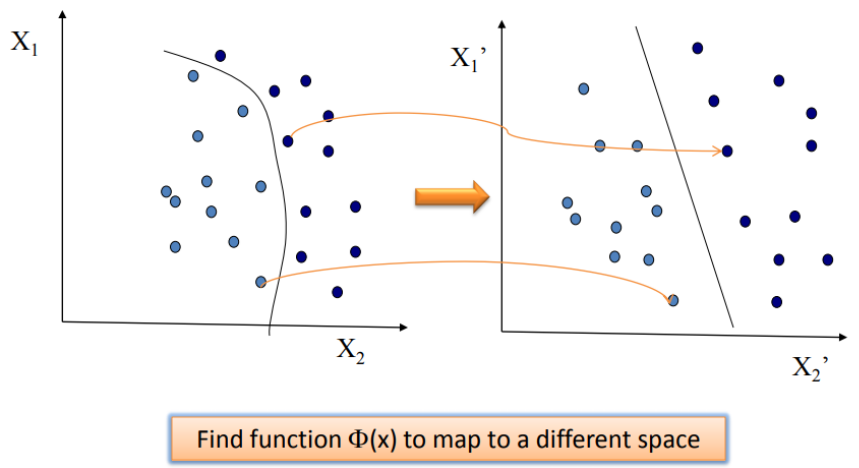

Figure 2. Linear Classifiers in High-Dimensional Spaces.

The data appear as $\phi(x)$ and weights $w$ are now weights in the new space. Furthermore, If $\phi(x)$ is very high dimensional, the explicit mapping is expensive.

\section{The kernel trick solution to simplify calculations:}

As long as mapping data to a high-dimensional space is so expensive, as an alternative solution, it come the kernel trick solution that uses the kernel function, defined as follows :

$$
K\left(x_{i}, x_{j}\right)=\phi\left(x_{i}\right)^{T} . \phi\left(x_{j}\right)
$$

With kernel trick solution, the computation of the separating hyperplane don't requires the knowledge of $\phi$, but only of $\mathrm{K}$.

Thus, it is possible to use any kernel function to redescribe in a larger dimension space. a presentation of the used kernel functions in this paper has been given in following section. We note that each kernel function has its advantages and disadvantages. It is therefore up to the user to choose the kernel, and the parameters of this kernel, which corresponds to his problem.

To summarize, the four great ideas behind the kernel trick solution are : 
- The data described in the entry space $\mathrm{E}$ are projected in a redescription space $\mathrm{E}$ '.

- Linear regularities are sought in this space E'.

- The Search algorithms don't need to know the coordinates of the projections of the data in E', but only their scalar products.

- These scalar products can be efficiently calculated through the use of kernel functions.

\subsection{Proposed SVM-based Classifier Description}

In this paper, we propose a new GNSS LOS/Multipath signal classifier based on the SVM and the fusion of information provided by the RHCP and LHCP antennas.

We present two signal reception state, namely, LOS and Multipath reception. The LOS signal transmitted by satellites is right-hand circular polarized (RHCP)[22]. When the signal is reflected, with an odd reflection number, the received signal become left-hand circular polarized (LHCP) [23].

The schematization of the proposed SVM-based classifier system is given in figure 3 .

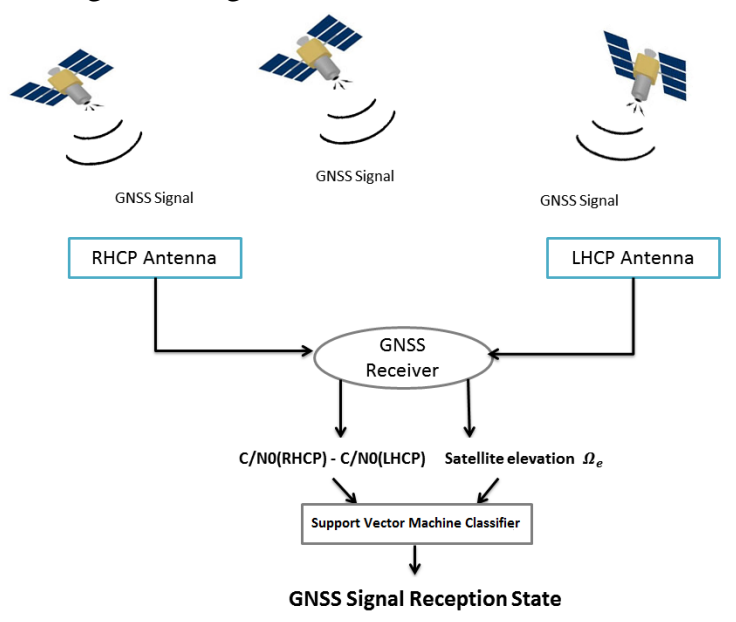

Figure 3. Global schematic set-up of the proposed SVM classifier system.

The SVM-based classifier has two inputs, namely, C/NO-R-L and satellite elevation, provided by RHCP and LHCP antennas, and one output is the "SRS" which indicate the signal reception state (Multipath or LOS signal).

\section{GNSS LOS/Multipath Signal Classification}

Our proposed SVM classifier is a system based on the SVM defined as a supervised technique of machine learning. At the heart of a SVM-based system exists a Training data set. The proposed SVM classifier aims to learn a model from the training data set, where the resulted model will be used to detect the GNSS signal reception state. To construct our training data set, we have defined a decision rules and the boundary values that characterizes each class (LOS or Multipath) from several experimental studies done in real environments. The decision rules are :
- If the direct signal is blocked and only reflected signals are being received, then the signal received by the RHCP antenna will be multiplied by an attenuation coefficients, which their value are less than 1 .

- If the LHCP antenna can not track the incoming signal, the received signal can be considered as a direct signal.

- If the RHCP antenna can not track the incoming signal, the signal can be considered as a reflected signal.

- The C/NO-R-L based thresholds for Multipath signals are inspired from Ziyi Jiang and Paul D. Groves study [16].

- When the signal is at high elevation, the received signal will be Weakly attenuate.

- $\mathrm{N}$ reflections has been considered, where $\mathrm{N}$ is an odd number.

- The delay dependent carrier phase between the RHCP and LHCP signal is null.

\subsection{SVM Classifier Design}

The architecture of the proposed SVM-based classifier system for GNSS Multipath detection is shown in figure 4. The main inputs of our SVM-based classifier is the training data set. Therefore, to construct our data set, we have used the results of the experimental studies and the decision rules. Consequently, a data set about 100 observations (LOS and Multipath) is constructed (See figure 5).

\subsection{Hardware and Data Acquisition}

To test the efficiency of the proposed SVM-based classifier, real GPS signals data are used.

The experimental data are acquired in real environment (See figure 6), Calais, France, in collaboration with LISIC Laboratory at the University of the Littoral Opal Coast (ULCO).

\subsubsection{Training Step}

The Training step aims to learn a model from the input data set. In this work, four kernel functions are used : Linear, Gaussian, Quadratic and Cubic.

\section{Model 1 : Linear SVM}

The Linear kernel function is the simplest kernel function. It is given by the inner product $\left\langle x_{i}, x_{j}>\right.$ plus an optional constant $\mathrm{c}$.

Therefore, the Kernel algorithms using a linear kernel are often equivalent to their non-kernel counterparts.

The linear kernel function can be written as follows :

$$
k\left(x_{i}, x_{j}\right)=x_{i}^{T} x_{j}+c,(c \text { is a constant })
$$

By applying our training data set, the resulting trained model is presented as follows : 


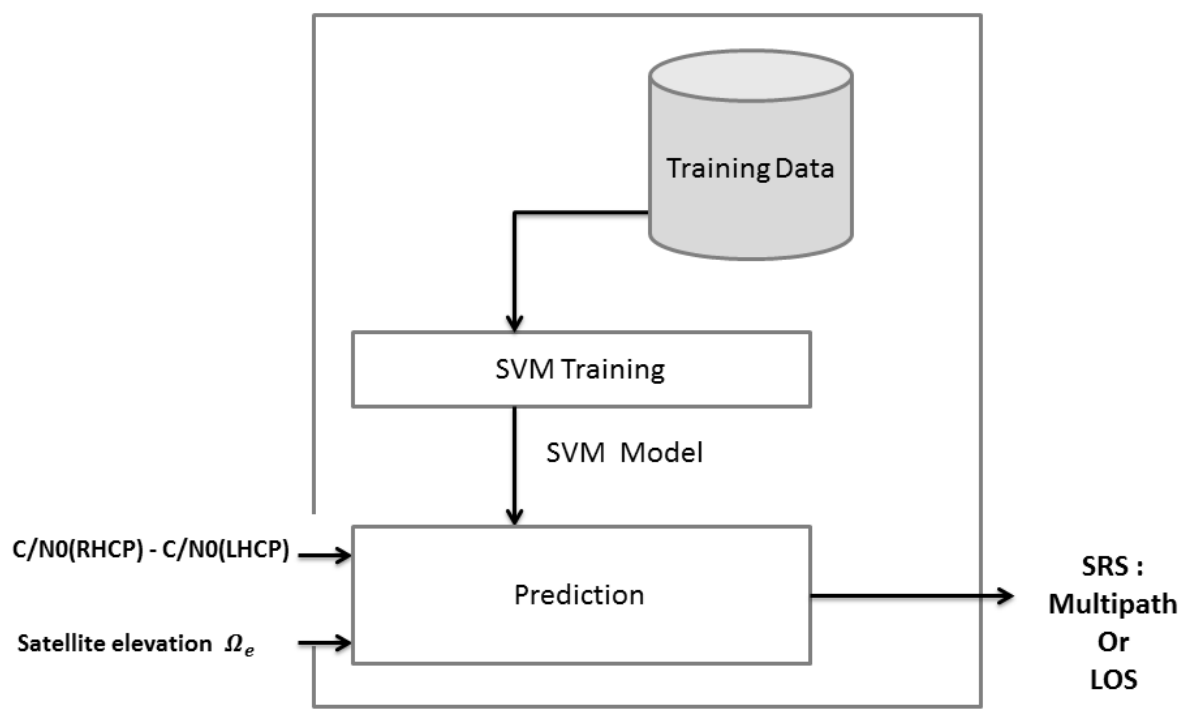

Figure 4. Conceptual structure of our SVM classifier system

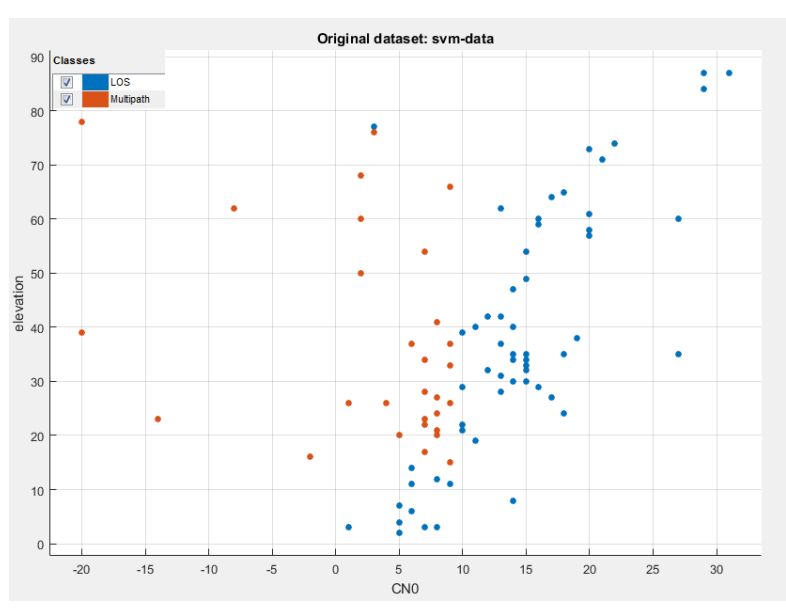

Figure 5. Training Data Set.

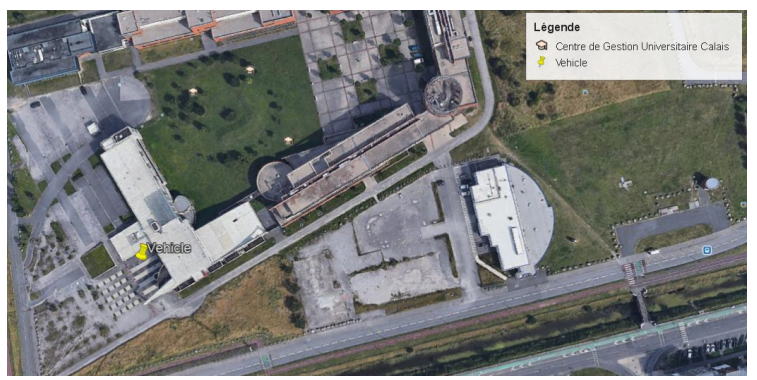

Figure 6. The data collection point at the park of the University Littoral Opal Coast, Calais, France

Furthermore, the model confusion matrix of the resulting SVM model of linear kernel function is presented in figure 7.

From figure 7, we show that we have 70 LOS observations, 68 of them are correctly learned and 2 are incorrectly learned (considered as Multipath). for the Multipath ob-

\begin{tabular}{|c|c|}
\hline \multicolumn{2}{|c|}{ Resulting Trained model } \\
\hline Accuracy & $85 \%$ \\
\hline Prediction speed & $8000 \mathrm{obs} / \mathrm{sec}$ \\
\hline Training time & $0.31936 \mathrm{sec}$ \\
\hline \multicolumn{2}{|c|}{ Classifier characteristics } \\
\hline Preset & Linear SVM \\
\hline Kernel function & Linear \\
\hline Kernel scale & automatic \\
\hline Box constraint level & 1 \\
\hline Multiclass method & One-vs-One \\
\hline Standardize data & true \\
\hline
\end{tabular}

Table 1. SVM using Linear kernel.

servations, we have 30,13 of them are incorrectly learned and 17 are correct.

From the obtained results, the error rate for signals classification using Linear SVM model is $15 \%$, hence the necessity to test another kernel function.

\section{Model 2 : Gaussian SVM}

The Gaussian kernel function of SVM is an example of radial basis function kernel. It can be written as :

$$
k\left(x_{i}, x_{j}\right)=\exp \left(-\frac{\left\|x_{i}-x_{j}\right\|^{2}}{2 \sigma^{2}}\right)
$$

Alternatively, it could also be expressed by :

$$
k\left(x_{i}, x_{j}\right)=\exp \left(-\sigma\left\|x_{i}-x_{j}\right\|^{2}\right)
$$

Where $\sigma$ must be carefully tuned, because its plays a major role in the performance of the kernel.

So, by applying our training data set, the resulting trained model is presented as follows : 


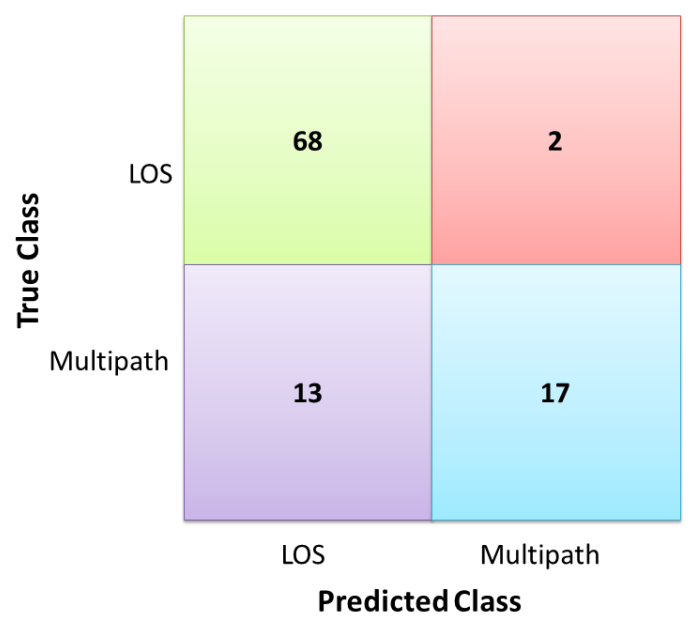

Figure 7. Linear SVM model confusion Matrix.

\begin{tabular}{|c|c|}
\hline \multicolumn{2}{|c|}{ Resulting Trained model } \\
\hline Accuracy & $93 \%$ \\
\hline Prediction speed & $7900 \mathrm{obs} / \mathrm{sec}$ \\
\hline Training time & $0.13598 \mathrm{sec}$ \\
\hline \hline \multicolumn{2}{|c|}{ Classifier characteristics } \\
\hline Preset & Medium Gaussian SVM \\
\hline Kernel function & Gaussian \\
\hline Kernel scale & 1.4 \\
\hline Box constraint level & 1 \\
\hline Multiclass method & One-vs-One \\
\hline Standardize data & true \\
\hline
\end{tabular}

Table 2. SVM using Gaussian kernel.

The model confusion matrix of the resulting SVM model of Gaussian kernel function is presented in figure 8. We show that we have 70 LOS observations, 68 of them are correctly learned and 2 are incorrectly learned (considered as Multipath). For the Multipath observations, we have 30,25 of them are correctly learned and 5 are incorrect.

From the obtained results, the error rate for signals classification using Gaussian SVM model is $7 \%$. We note that Gaussian kernel gives best results compared to Linear model. Furthermore, $7 \%$ of error is a no-negligible rate, hence the necessity to test another kernel function.

\section{Model 3 : Cubic SVM}

The Cubic polynomial kernel can be presented as :

$$
k\left(x_{i}, x_{j}\right)=\left(x_{i}^{T} x_{j}+1\right)^{3}
$$

So, by applying our training data set, the resulting trained model is presented as follows :

The model confusion matrix of the resulting SVM model of Cubic kernel function is presented in figure 9.

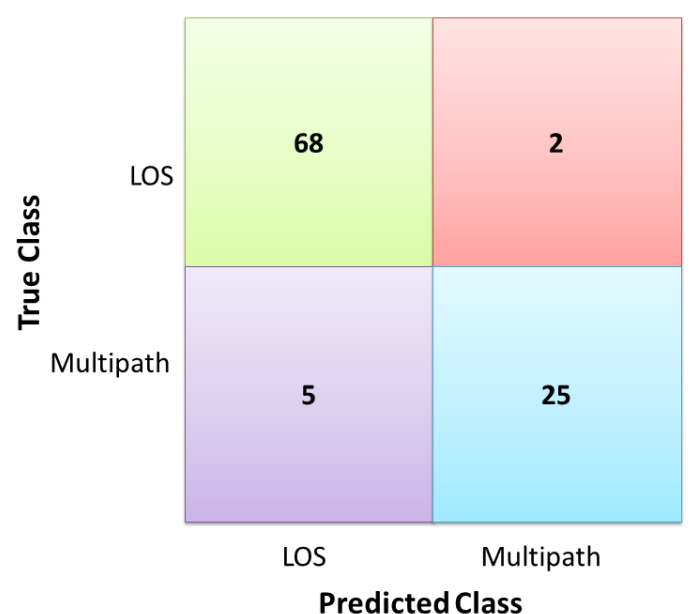

Figure 8. Gaussian SVM model confusion Matrix.

\begin{tabular}{|c|c|}
\hline \multicolumn{2}{|c|}{ Resulting Trained model } \\
\hline Accuracy & $89 \%$ \\
\hline Prediction speed & $8000 \mathrm{obs} / \mathrm{sec}$ \\
\hline Training time & $0.183 \mathrm{sec}$ \\
\hline \hline \multicolumn{2}{|c|}{ Classifier characteristics } \\
\hline Preset & Cubic SVM \\
\hline Kernel function & Cubic \\
\hline Kernel scale & Automatic \\
\hline Box constraint level & 1 \\
\hline Multiclass method & One-vs-One \\
\hline Standardize data & true \\
\hline
\end{tabular}

Table 3. SVM using Cubic kernel.

We show that we have 70 LOS observations, 64 of them are correctly trained and 6 are incorrectly learned (considered as Multipath).

For the Multipath observations, we have 30, 5 of them are incorrectly trained and 25 are correctly trained.

From the obtained results, the error rate for signals classification using SVM Cubic Kernel is $11 \%$, hence the necessity to test another kernel function.

\section{Model 4 : Quadratic SVM}

The Quadratic kernel function is less computationally intensive than the Gaussian kernel function and can be used as an alternative solution when using the Gaussian kernel becomes too expensive. The Quadratic function can be written as follows:

$$
k\left(x_{i}, x_{j}\right)=1-\frac{\left\|x_{i}-x_{j}\right\|^{2}}{\left\|x_{i}-x_{j}\right\|^{2}+c}
$$

By applying our training data set, the resulting trained model is presented as follows : 


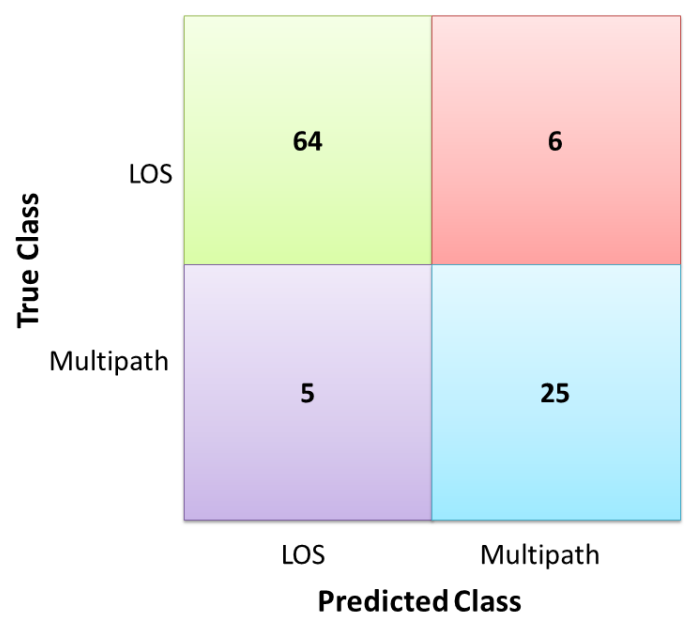

Figure 9. Cubic SVM model confusion Matrix.

\begin{tabular}{|c|c|}
\hline \multicolumn{2}{|c|}{ Resulting Trained model } \\
\hline Accuracy & $92 \%$ \\
\hline Prediction speed & $7400 \mathrm{obs} / \mathrm{sec}$ \\
\hline Training time & $0.14757 \mathrm{sec}$ \\
\hline \hline \multicolumn{2}{|c|}{ Classifier characteristics } \\
\hline Preset & Quadratic SVM \\
\hline Kernel function & Rational Quadratic \\
\hline Kernel scale & Automatic \\
\hline Box constraint level & 1 \\
\hline Multiclass method & One-vs-One \\
\hline Standardize data & true \\
\hline
\end{tabular}

Table 4. SVM using rational Quadratic kernel.

Furthermore, the model confusion matrix of the resulting SVM model of Quadratic kernel function is presented in figure 10.

From figure 10, we show that we have 70 LOS observations, 67 of them are correctly learned and 3 are incorrectly trained. For the Multipath observations, we have 30, 25 of them are correctly learned and 5 are incorrect.

From the obtained results, the error rate for GNSS signals classification using SVM Quadratic Kernel is 8\%. Consequently, we can conclude that the Quadratic kernel function has given best classification accuracy compared to $\mathrm{Cu}$ bic and Linear kernel.

\section{SVM Trained model Discussion}

From the resulting SVM trained model using the Gaussian, Cubic, Quadratic and Linear kernels, we have shown that the Gaussian kernel has given the best signal classification accuracy.

Therefore, for the LOS observations, the trained models using Linear and Gaussian kernels have given the same results (68 observations are correctly trained from 70 LOS observations). Furthermore, the Gaussian, Cubic and Quadratic kernels have given the same results of Multi-

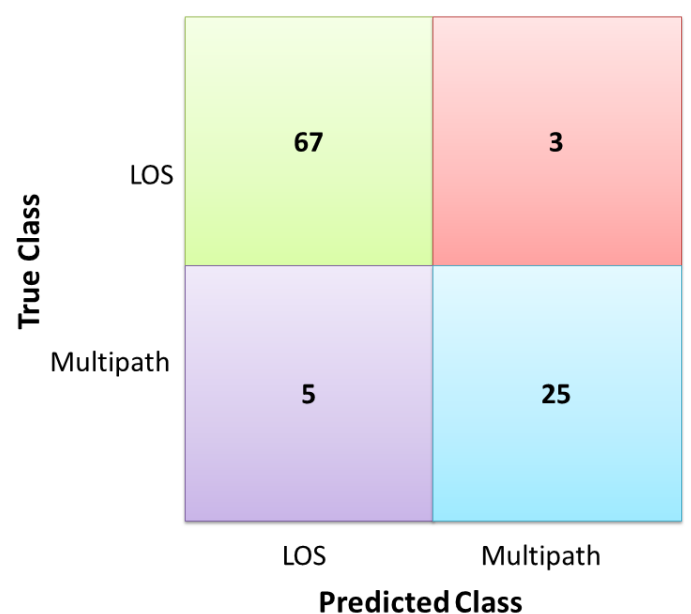

Figure 10. Quadratic SVM model confusion Matrix.

path observations (25 Multipath observations are correctly trained from 30 Multipath observations).

\subsubsection{Prediction Step}

In this step, we used a new data set composed by new observations(each line is a couple of C/NO-R-L and satellite elevation) and we apply the trained models resulting in the "Training step".

The table 5 shows the results of the prediction step by applying the proposed SVM-based classifier system. From table 5, we can confirm that the SVM-based model using Gaussian kernel function has given the best results over Cubic, Quadratic and Linear kernels. Furthermore, our objective is to classify the incoming GNSS signals from satellites. As a results, the efficiency of the proposed SVM-based classifier system to detect the signal reception state has been clearly demonstrated. We have obtained the same the expected results.

\section{Conclusions}

In this paper, we have proposed a new technique to classify GNSS signal reception state using the fusion of information provided by two antennas (RHCP and LHCP) and a machine learning technique.

The GNSS signals classification is characterized by two measures, satellite elevation and C/NO-R-L which is the difference between the C/NO from RHCP and LHCP antennas. As long as the classification measures are uncertain and imprecise, a SVM-based approach has been applied. The SVM-based classifier uses a data set of two inputs (C/NO-R-L and satellite elevation). The trained data set is constructed by observations from real GPS signals. The classification Learner toolbox of Matlab has used to design our SVM-based classifier, with training and prediction steps.

Using real GPS signals, the efficiency of the SVM-based classifier for GNSS signals classification has been shown. 


\begin{tabular}{|c|c|c|c|c|c|}
\hline \hline \multicolumn{2}{|c|}{ Inputs of SVM-based classifier system } & \multicolumn{4}{|c|}{ SVM Prediction using different kernels } \\
\hline C/NO-R-L & Satellite elevation $\Omega_{e}$ & Gaussian & Cubic & Quadratic & Linear \\
\hline 11.99 & 33.18 & LOS & LOS & LOS & LOS \\
\hline 14.23 & 33.94 & LOS & LOS & LOS & LOS \\
\hline 51 & 60.31 & LOS & LOS & LOS & LOS \\
\hline 12.63 & 31.79 & LOS & LOS & LOS & LOS \\
\hline 18.05 & 65.94 & LOS & Multipath & Multipath & LOS \\
\hline 20.62 & 58.39 & LOS & Multipath & LOS & LOS \\
\hline 18 & 35.88 & LOS & LOS & LOS & LOS \\
\hline 17.29 & 87.68 & LOS & Multipath & Multipath & LOS \\
\hline 13.44 & 37.82 & Multipath & Multipath & Multipath & Multipath \\
\hline 18.49 & 70.25 & LOS & LOS & LOS & Multipath \\
\hline 15.77 & 79.01 & LOS & LOS & LOS & Multipath \\
\hline 6.69 & 17.31 & Multipath & Multipath & Multipath & Multipath \\
\hline 7.27 & 34.22 & Multipath & Multipath & Multipath & Multipath \\
\hline
\end{tabular}

Table 5. Signal reception state detection using the proposed SVM-based classifier system.

\section{Acknowledgments}

This work was financially supported by the National Center of Scientific and Technical Research (CNRST), Morocco.

We thank Mr Serge Reboul, Mr Georges Stienne and Mr Jean-Bernard Choquel from ULCO university (LISIC laboratory) for their collaboration in designing the proposed GNSS classifier based on RHCP and LHCP antennas, and their assistance in data acquisition.

\section{References}

[1] A. Rabaoui, N. Viandier, E. Duflos, J. Marais, P. Vanheeghe, Dirichlet process mixtures for density estimation in dynamic nonlinear modeling: Application to GPS positioning in urban canyons (IEEE, 2012), Vol. 60, pp. 1638-1655

[2] L. Wang, P.D. Groves, M.K. Ziebart, Urban positioning on a smartphone: real-time shadow matching using GNSS and 3D city models (The Institute of Navigation, 2013)

[3] L. Wang, P.D. Groves, M.K. Ziebart, GNSS shadow matchin: Improving urban positioning accuracy using a 3D city model with optimized visibility scoring scheme (Wiley Online Library, 2013), Vol. 60, pp. 195-207

[4] L. Wang, P.D. Groves, M.K. Ziebart, Smartphone shadow matching for better cross-street gnss positioning in urban environments (Cambridge Univ Press, 2015), Vol. 68, pp. 411-433

[5] Y. Gu, L.T. Hsu, S. Kamijo, GNSS/On-Board Inertial Sensor Integration with the Aid of 3D Building Map for Lane-Level Vehicle Self-Localization in Urban Canyon (IEEE, 2015)

[6] B. Guermah, T. Sadiki, H. El Ghazi, Fuzzy logic approach for GNSS signal classification using RHCP and LHCP antennas, in Ubiquitous Computing, Electronics and Mobile Communication Conference
(UEMCON), 2017 IEEE 8th Annual (IEEE, 2017), pp. 203-208

[7] B. Guermah, T. Sadiki, H. El Ghazi, S. Reboul, E. Ahouzi, A Map-Matching Based Approach to Compute and Modelize NLOS and Multipath Errors for GNSS Positioning in Hard Areas (2017), Vol. 13, pp. 256-269

[8] A. Pirsiavash, A. Broumandan, G. Lachapelle, Characterization of signal quality monitoring techniques for multipath detection in GNSS applications (Multidisciplinary Digital Publishing Institute, 2017), Vol. 17, p. 1579

[9] G. Bassma, S. Tayeb et al., GNSS Positioning Enhancement Based on NLOS Multipath Biases Estimation Using Gaussian Mixture Noise, in International Journal of Mobile Computing and Multimedia Communications (IJMCMC) (IGI Global, 2018), Vol. 9, pp. 21-39

[10] N. Crocetto, S. Ponte, G. Puglianoc, L. Savino, Fuzzy-Logic Based Methodologies for Mobile Mapping: Enhancing Positioning Accuracy of GPS/GNSS Measurements (2007), Vol. 36

[11] C.J. Lin, Y.Y. Chen, F.R. Hang, Fuzzy processing on GPS data to improve the position accuracy, in Fuzzy Systems Symposium, 1996. Soft Computing in Intelligent Systems and Information Processing., Proceedings of the 1996 Asian (IEEE, 1996), pp. 557-562

[12] A. Ghalehnoe, K. Mohammadi, M. Mosavi, Improve determining the location of a moving body on map by low cost GPS receiver, in Research and Development, 2002. SCOReD 2002. Student Conference on (IEEE, 2002), pp. 242-244

[13] J. Wang, Y. Gao, Evaluating the accuracy of GPS positions under severe signal-degradation using adaptive-network-based fuzzy inference systems (ANFIS), in 50th CASI Annu. General meeting and Conf., Canadian Aeronautics and Space Inst., Montréal, Quebec, Canada (2003) 
[14] C. Cheng, J.Y. Tourneret, Q. Pan, V. Calmettes, Detecting, estimating and correcting multipath biases affecting GNSS signals using a marginalized likelihood ratio-based method (Elsevier, 2016), Vol. 118, pp. 221-234

[15] J.H. Wang, Y. Gao, Identification of GPS positioning solutions deteriorated by signal degradations using a fuzzy inference system (Springer, 2004), Vol. 8, pp. 245-250

[16] Z. Jiang, P.D. Groves, NLOS GPS signal detection using a dual-polarisation antenna (Springer, 2014), Vol. 18, pp. 15-26

[17] M. Socharoentum, H.A. Karimi, Y. Deng, A machine learning approach to detect non-line-of-sight GNSS signals in Nav2Nav (2016)

[18] L.T. Hsu, GNSS multipath detection using a machine learning approach, in Intelligent Transportation Systems (ITSC), 2017 IEEE 20th International Conference on (IEEE, 2017), pp. 1-6
[19] Z.M. Miao, L.W. Zhao, W.W. Yuan, F.L. Jin, Application of One-Class Classification in NLOS Identification of UWB Positioning, in Information System and Artificial Intelligence (ISAI), 2016 International Conference on (IEEE, 2016), pp. 318-322

[20] S. Tong, D. Koller, Support vector machine active learning with applications to text classification (2001), Vol. 2, pp. 45-66

[21] M. Frank, P. Wolfe, An algorithm for quadratic programming (Wiley Online Library, 1956), Vol. 3, pp. 95-110

[22] K. Zhang, B. Li, X. Zhu, H. Chen, G. Sun, NLOS Signal Detection Based on Single Orthogonal DualPolarized GNSS Antenna (Hindawi Publishing Corporation, 2017), Vol. 2017

[23] M. Brenneman, J. Morton, F. van Grass, C. Yang, Mitigation of GPS multipath using polarization and spatial diversities (2008) 